control group of 334 women who did not develop CHD. Blood samples were obtained from cases and controls at baseline and after a 6-h fast, and concentrations of serum adiponectin, C-reactive protein, insulin, glucose, total cholesterol, HDL cholesterol and LDL cholesterol and triglyceride levels were measured.

Adiponectin was inversely correlated with insulin, glucose, C-reactive protein and triglyceride levels in both the case and control groups, however, no association was found between adiponectin levels and incident CHD.

The authors conclude that it is premature to associate adiponectin with the risk of developing CHD, and call for further prospective studies to determine the effect of this hormone on CHD and establish whether a difference between men and women actually exists.

Marie Lofthouse

Original article Lawlor DA et al. (2005) Plasma adiponectin levels are associated with insulin resistance but do not predict future risk of coronary heart disease in women. J Clin Endocrinol Metab [doi:10.1210/jc.2005-0825]

\section{Thyroid-stimulating hormone modulates gene expression in vivo}

Complex mechanisms regulate thyroid physiology. Although some information is available, the data from in vitro studies on expression of individual genes are inconsistent and difficult to extrapolate to the human thyroid. A prospective single-center study has now demonstrated the importance of human TSH in the differential expression of thyroid-specific genes in vivo.

The authors measured gene expression in both normal and nodular thyroid specimens from 39 patients undergoing thyroidectomy. In 17 patients (Group 1), serum TSH levels were normal and surgery was performed soon after diagnosis. The remaining 22 patients (Group 2) had undergone TSH suppression for $\geq 6$ months, resulting in a TSH level $<0.5 \mathrm{mU} / \mathrm{l}$.

In normal tissue from Group 2, levels of sodium-iodine symporter SLC5A5 (NIS) and apical iodide transporter SLC5A8 (AIT) gene expression were lowest and thyroglobulin gene expression was highest. Comparison of normal tissue from the two groups showed reduced transcription of the SLC5A5 and thyroperoxidase genes in Group 2, along with minor reductions in transcription of SLC5A8, thyroglobulin and the transcription factor
PAX8. Transcription of the $\mathrm{TSH}$ receptor $T S H R$, transcription factor TTF1 and pendrin SLC26A4 genes was unaltered. After exclusion of patients with malignancy, SLC5A5 mRNA expression was lower in nodular than in normal tissue from Group 1; the same was not seen in Group 2.

Results suggest that TSH is involved in the transcription of most thyroid-specific hormones, with expression of some being completely dependent on its presence. Understanding of the role of $\mathrm{TSH}$ might be enhanced through gene-array or proteomic studies.

Rebecca Doherty

Original article Bruno R et al. (2005) Modulation of thyroidspecific gene expression in normal and nodular human thyroid tissues from adults: an in vivo effect of thyrotropin. J Clin Endocrinol Metab [doi: 10.1210/jc.2005-0800]

\section{Parathyroid hormone followed by alendronate maintains BMD gains in osteoporosis patients}

The FDA recommends that parathyroid hormone (PTH), a drug administered to osteoporosis patients to increase bone mineral density (BMD), is taken for a maximum of 2 years. After discontinuation, some of the gains in BMD could be lost. According to a recent study, following PTH with the antiresorptive agent alendronate can maintain or increase these gains.

This controlled, double-blind, randomized, prospective study was an extension of a trial of previously untreated, postmenopausal women with osteoporosis. Results had shown that alendronate and full-length (amino acids 1-84) PTH combined therapy was ineffective. In this extension, patients who had already received PTH monotherapy for 1 year were randomized to a further year of oral alendronate $(n=59)$ or placebo $(n=60)$. Patients who had received aledronate plus PTH or alendronate alone underwent a further year of alendronate monotherapy $(n=59$ and $n=60$, respectively).

Over 2 years, patients who took PTH monotherapy followed by alendronate showed a $31 \%$ increase in volumetric spinal BMD, compared with only $14 \%$ in those who followed PTH with placebo. PTH followed by alendronate resulted in numerically greater (although not always significant) gains in areal BMD at trabecular-bone-rich sites at the spine and hip than alendronate alone; little difference 